\title{
ARTICLE
}

\section{Raman Imaging and Chemometrics Evaluation of Natural and Synthetic Beeswaxes as Matrices for Nanostructured Lipid Carriers Development}

\author{
Hery Mitsutake ${ }^{1,2 *}$ (D) Gustavo Henrique Rodrigues da Silva2 ${ }^{\text {ID }}$, Lígia Nunes de Morais \\ Ribeiro ${ }^{\text {ID }}$, Eneida de Paula2 iD, Ronei J. Poppi ${ }^{1}$ iD †, Douglas N. Rutledge ${ }^{3,4}$ (D), Márcia C. \\ Breitkreitz ${ }^{1}$ iD
}

${ }^{1}$ Department of Analytical Chemistry, Institute of Chemistry, University of Campinas (Unicamp), Rua Josué de Castro, s/n Cidade Universitária Zeferino Vaz, 13084-970, Campinas, SP, Brazil

${ }^{2}$ Department of Biochemistry and Tissue Biology, Institute of Biology, University of Campinas (Unicamp), Rua Monteiro Lobato, 255, 13083-862, Cidade Universitária Zeferino Vaz, Campinas, SP, Brazil

${ }^{3}$ Université Paris-Saclay, INRAE, AgroParisTech, UMR SayFood, 75005, Paris, France

${ }^{4}$ National Wine and Grape Industry Centre, Charles Sturt University, Wagga Wagga, Australia

† In memoriam (1961 - 2020)

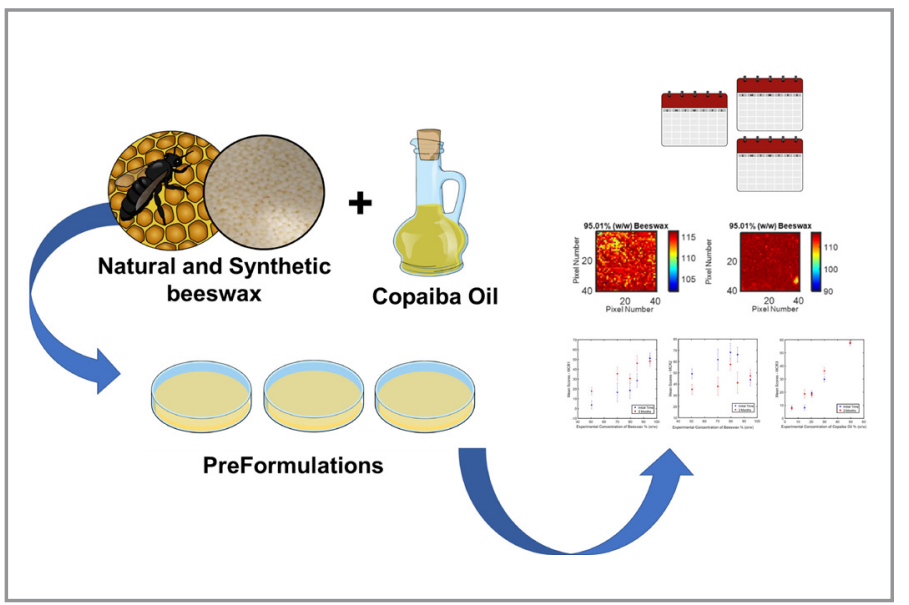

Beeswaxes are interesting solid lipids for the development of nanostructured lipid carriers (NLC), and their origin can be either natural or synthetic. Due to this difference, their performance should be distinct and unstable formulations can be generated. The objective of this work was to investigate miscibility and structural changes (polymorphism) in pre-formulations (blends of solid and liquid lipids) using synthetic and natural beeswaxes in combination with copaiba oil (a natural liquid lipid), in the concentration range of 5.0 to $50.0 \%(w / w)$. Raman spectra were acquired over a region of $4 \mathrm{~mm}^{2}$ (mapping mode), dead pixels were removed using Independent Components Analysis (ICA) and Multivariate Curve Resolution - Alternating Least Squares (MCR-ALS) was then used to generate the images. Samples were analyzed at the initial time and after 3 months, using the Distributional Homogeneity Index (DHI) and standard deviation of the histograms. The pre-formulation containing synthetic beeswax showed different structural forms before and after melting, and structural changes over time, depending on the amount of the liquid lipid incorporated. These results demonstrate how spectroscopic imaging techniques can be valuable in pharmaceutical development, as well as the importance of choosing the type and proportion of solid lipid to achieve stable NLC formulations. 
Keywords: beeswax, copaiba oil, DHI, MCR-ALS, ICA

\section{INTRODUCTION}

Beeswaxes (BW) are a source of lipids that can be used in the development of nanostructured lipid carriers (NLC) [1,2] and they can be of either natural or synthetic sources. Natural beeswaxes (nBW) are produced by the species Apis mellifera and Apis cerana. The composition of this product is variable, depending both on the bee species and the region of production. It is a complex mixture, composed of more than 300 components, divided into: hydrocarbons - mainly C27-C33 chains (12-16\%), free fatty acids with C24-C32 (12-14\%), free fatty alcohols - C28-C35 ( 1\%), monoesters and hydroxymonoesters - C40-C48 derived mainly from palmitic, 15-hydroxpalmitic and oleic acids (35-45\%) and complex esters (15-27\%) such as 15-hydroxy-palmitic acid or diols [3]. Synthetic BW (sBW) (CAS number 71243-51-1) are defined by the United States Environmental Protection Agency (EPA) as "the product of the complex reaction of mixtures of acids and alcohols that simulate the composition of natural BW. It consists mainly of alkyl esters - C18-C30 of fatty acids C16-C32, fatty acids - C16-C32, and alkanes - C22-C34" [4]. Copaiba (Copaifera officinalis) is a tree native to South America and the oil-resin produced by it is known for its medicinal properties (e.g., anti-inflammatory, antiseptic and antitetanic). Copaiba oil is rich in sesquiterpene hydrocarbons, mainly $\beta$-caryophyllene which is responsible for its biological activites $[5,6]$. Other compounds found in this oil are diterpene acids, copalic acid, hardwickiic acid, allo-aromadendrene, germacrene B, $\beta$-bisabolene, $\alpha$-cadinene, $\gamma$-cadinene, trans- $\alpha$-bergamotene and $\alpha$-humulene [7].

Attama and coworkers characterized mixtures of $\mathrm{nBW}$ and cocoa oil for NLC production and demonstrated that formulations with 50 and $75 \%$ (w/w) of oil could be used for the development of lipid nanoparticles [8]. Ribeiro et al. developed formulations of nBW with copaiba oil, which showed good physicochemical stability for 12 months, with prolonged in vitro release and in vivo effect of lidocaine [1]. Lima et al. developed solid lipid nanoparticles (SLN) and NLC formulations with beeswax and stearic acid for the delivery of tacrolimus. BW allowed formulations with high drug upload without stability issues [9]. The polymorphism of solid excipients used in the development of SLN and NLC is one of the major problems that can promote shelf-instability in these formulations, probably due to the expulsion of the Active Pharmaceutical Ingredient (API) from the nanoparticles. However, this characteristic does not hamper the use of a given excipient, but rather indicates that attention with miscibility issues should be taken at the very early stages of formulation. One example is the cocoa and shea butter used on NLC by Ribeiro et al. [1].

Beeswax is a solid lipid that can show polymorphism. Therefore, the characterization of this raw material is essential prior to its use in pharmaceutical formulations [10]. In this sense, the use of Raman imaging in the early stages of pharmaceutical development can give much information, since this technique is sensitive to both chemical and physical changes in the material. Using this technique, mixtures of solid and lipid excipients (which will here be called 'pre-formulations') can be evaluated prior to their mixing with surfactants and other excipients, with the aim of forecasting stability issues. This approach allows a rational screening of excipients during the NLC development. Thus, it is possible to avoid instabilities and waste of money during this step. We have recently demonstrated how this tool can be useful for evaluating the miscibility of excipients such as Capryol ${ }^{\circledR}$ 90, Precirol ATO5 ${ }^{\circledR}$, Dhaykol $6040^{\circledR}$ and cetyl palmitate by combining Raman imaging with classical least squares (CLS). In this previous work, the standard deviation of the histograms of pixel was used to evaluate the miscibility. However, this parameter can also be assessed with the help of the distributional homogeneity index (DHI), a technique based on macropixel analysis developed by Sacré et al. [11]. DHI does not require a calibration model and may be used to compare the homogeneity of different formulations. Also, it avoids the subjective evaluation through the use of the kurtosis of the histograms.

It should be noted that defects may occur in vibrational spectroscopy images, due to instrumental, sample presentation, or radiation issues. These problems generate defects: the affected pixels being called dead/bad pixels, non-informative background or outliers, which can all affect the performance of models for identification, classification and quantification [12]. Therefore, it is necessary to eliminate these defects before applying a chemometric method. In some cases, these pixels can be purged with adequate 
preprocessing or replaced by the mean or median of the neighbors. Since many of these defects are physical and independent phenomena, independent components analysis (ICA) can be used to correct these defective pixels $[13,14]$. ICA is a Blind Source Separation (BSS) method based on Central Limit Theorem that states that mixtures of several independent source signals should give intensity distributions which are more gaussian than those of the individual source signals.

Therefore, the objective of this work was to evaluate the structural transformations and miscibility between synthetic and natural beeswaxes and copaiba oil - in the range of 5.0-50.0\% (w/w) - using Raman mapping and chemometrics. In a first step, the two different beeswaxes were analyzed by X-ray diffraction, differential scanning calorimetry (DSC) and Raman spectroscopy. In the second step, ICA was used to eliminate artefacts in the spectral images. Afterwards, chemical maps were built using MCR-ALS scores and their homogeneity was evaluated using the standard deviation of histograms (STD) and the DHI criterion.

\section{MATERIALS AND METHODS Materials}

Natural beeswax was donated from GM Ceras (Brazil) while synthetic beeswax (Meghwax SEW 200) was provided by Megh Industria e Comércio (Brazil). Copaiba oil (Copaifera officinalis) was purchased from Phytoterápica Cosméticos (Brazil).

\section{DSC Curves}

A TA Instruments equipment (model Q100) was used in the temperature range of 20 to $250{ }^{\circ} \mathrm{C}$, with heating rate of $10^{\circ} \mathrm{C} / \mathrm{min}$ and inert atmosphere (argon $\left.50 \mathrm{~mL} / \mathrm{min}\right)$. Beeswax samples (3-14 mg) were weighed and placed in hermetic aluminum pans for the analysis.

\section{$X-R a y$ Diffraction}

The beewaxes used in pre-formulations were analyzed by X-ray diffraction before and after melting. A Shimadzu X-ray diffractometer (model XRD7000) was used under the following conditions: Cu radiation $(1.54060 \mathrm{~A})$, voltage of $40 \mathrm{kV}$, current of $30.0 \mathrm{~mA}, 2$ degrees $2 \theta /$ minute, in the range of 5.0 to 50.0 degrees $2 \theta$.

\section{Sample Preparation and Raman Mapping}

Five pre-formulations were developed, varying copaiba oil from 5.0-50.0 (\% w/w). The samples were prepared by heating $\left(10^{\circ} \mathrm{C}\right.$ above the melting point of the beeswax) and copaiba oil was added with stirring until a visually homogeneous mixture was obtained. The samples were cooled to room temperature $\left(25 \pm 2{ }^{\circ} \mathrm{C}\right)$ in an aluminum cell and an area of $2.0 \times 2.0 \mathrm{~mm}\left(4 \mathrm{~mm}^{2}\right)$ was mapped using a Raman Station 400 (Perkin Elmer, CT, USA). A $785 \mathrm{~nm}$ laser of was used as an excitation light and power of $100 \mathrm{~mW}$.

The exposure time was 3s/pixel and each spectrum was the average of 2 exposures. The step size was $50 \mu \mathrm{m}$ and the spectral range $600-3200 \mathrm{~cm}^{-1}$ with a resolution of $4 \mathrm{~cm}^{-1}$. Each sample map $\left(4 \mathrm{~mm}^{2}\right)$ generated a cube of data with dimensions of $40 \times 40 \times 651$, where 40 was the number of pixels at $x$ and $y$ axis and 651 the number of spectral variables. The total mapping time for each image acquisition was 4 hours. The flasks were sealed and stored in the dark. Raman images of all samples were obtained at the initial time and 3 months after preparation. The period of 3 months was chosen based on recommendations of ICH Q1A(R2) guideline. Three months is the time that for preformulation stability studies are normally carried out by pharmaceutical companies [15].

\section{Data Processing}

Spikes from Raman spectra were excluded using an algorithm developed by Sabin and co-workers [16]. The spectral range of $1804-1044 \mathrm{~cm}^{-1}$ was selected. The data cube was unfolded to a 2D (NM $\times$ $\lambda$ ) matrix, where $M$ is number of pixels on the $x$ axis, $N$ is the number of pixels on the $y$ axis and $\lambda$ is the number of spectral variables. The spectral baseline was corrected by asymmetric least squares [17] and unit vector normalization. 


\section{Chemometric Analysis}

Independent Components Analysis

ICA is a BSS method proposed by Jutten and Herault [18] to extract pure underlying signals (source signals) from a set of mixed signals (Equation 1):

$$
\boldsymbol{X}=\boldsymbol{A S}
$$

where $\mathbf{X}(n \times \lambda)$ is the data matrix, $\mathbf{S}(k \times \lambda)$ is a matrix of independent source signals, $\mathbf{A}(n \times k)$ is a matrix of mixing coefficients or proportions of the pure signals, $k$ is the number of independent components (IC), $\lambda$ is the number of variables (wavenumbers) and $n$ is the number of samples/pixels.

ICA assumes that the source signals and their proportions in the analyzed mixtures are unknown, and it aims to extract them by using the criterion of maximum independence among the source signals. In this work the Joint Approximate Diagonalization of Eigenmatrices (JADE) algorithm [19] was used. This algorithm has the advantage of being deterministic, i.e., not subject to convergence problems since initial random estimates are not necessary [20]. An important parameter during the construction of the ICA model is the choice of the number of components since for a model with $A$ factors and one with $A+1$ factors, some ICs do not have the same index in both models, and/or do not have the same signal or are simply different. In this work the Durbin-Watson criterion was used [21]. After the application of ICA with the optimal number of ICs, one IC was identified as containing the information concerning the physical defect, which was removed from the data matrix using Equations 2 and 3:

$$
\begin{gathered}
\boldsymbol{I C}_{\text {physical defect }}=\boldsymbol{a}_{\text {physical defect }} * \boldsymbol{S}_{\text {physical defect }}{ }^{T} \\
\boldsymbol{X}_{\text {corrected }}=\boldsymbol{X}_{\text {preprocessed }}-\boldsymbol{I C}_{\text {physical defect }}
\end{gathered}
$$

where $\boldsymbol{a}_{\text {physical defect }}$ refer to the ICA proportions, $\boldsymbol{s}_{\text {physical defect }}$ is the source signal of the IC with physical information and $\boldsymbol{X}_{\text {corrected }}$ represents the matrix after subtraction of this information.

\section{Multivariate Curve Resolution - Alternating Least Squares}

MCR-ALS is the most popular algorithm for multivariate curve resolution, based on Equation 4:

$$
X=C S_{1}^{T}+E
$$

where $\mathbf{C}$ is the concentration profile $(\mathrm{MN} \times \mathrm{G}), \mathbf{S}_{1}$ the spectral profile $(\lambda \times G)$ and $\mathbf{E}$ is residual matrix (MN $x \lambda$ ) for $\mathbf{G}$ components. $\mathbf{C}$ and $\mathbf{S}_{1}$ are iteratively calculated until convergence. This iterative aspect of the algorithm presents advantages as it is possible to model spectral profiles with small changes that could be caused by interactions. The number of components was chosen based on singular value decomposition (SVD). The constraints of non-negativity of both spectral and concentration profiles were used in this work. Equality constraint was also used for copaiba oil spectrum in the model for natural BW. The pure excipient spectra were used as initial estimates of $\mathbf{S}$. For the MCR analysis the MCR_gui version 2 toolbox [22] was used.

\section{Distributional Homogeneity Index}

$\mathrm{DHI}$ is a subsampling technique based on analysis of macropixels. A macropixel is defined as "square cluster of neighboring pixels with an intensity value equal to the average value of the included pixels". In the first step, the chemical map was sampled for all possible macropixels of size 22 . In the next step, all macropixels of size 33 were evaluated. This continues until the macropixel size was equal to whole chemical map size (continuous-level moving block). At each step, the standard deviation of the macropixels was 
calculated and plotted vs. the macropixel size to generate the homogeneity curve $[11,23]$. Afterwards the chemical map was randomized several times (usually 50 to 100) and a homogeneity curve of each map was calculated. DHI was obtained from the ratio of the area under the homogeneity curve (AUC) of the original map and the AUC of the randomized map. So, the greater the $\mathrm{DHI}$ values, the more heterogenous the sample [11]. All calculations were done using Matlab version 8.3 (Mathworks Inc., Natick MA, USA).

\section{RESULTS AND DISCUSSION}

\section{Structural Characterization of the Beeswaxes}

Before the construction of the models, Raman spectra, DSC curves and X-ray diffractograms of the beeswaxes were acquired, before and after the fusion (Figure 1). Synthetic wax displayed structural changes after melting, which was not observed for nBW. X-ray diffraction analysis was performed on natural and synthetic BW in order to verify the alteration in the crystalline structure, (Figure 1a and 1b). While there was a clear loss of structure in sBW (due to peak disappearance), no significant changes (except for a slight shift to higher $2 \theta$, from 21.0 to 24.0 , after melting) were observed in the natural sample. A very weak peak observed at $5.7^{\circ}$ in the sBW diffractogram before melting - corresponding to second or higher order of the long spacing reflection of the diester fraction (monoclinic structure) - disappeared after the melting process. Higher intensities localized at 21.3 to 24.2 in both samples were related to the orthorhombic structure of hydrocarbon/monoester fractions [10] and these peaks seem to be characteristic of beeswax [2,9]. In the DSC curves the polymorphism of sBW was identified by the decrease in the melting point $\left(63.76\right.$ to $\left.62.06^{\circ} \mathrm{C}\right)$ and the appearance of a new peak at $54.5^{\circ} \mathrm{C}$ (Figure $\left.1 \mathrm{C}\right)$. In $\mathrm{nBW}$, the curve does not change significantly.

This change in sBW was also observed in the Raman spectra due to the disappearance of the peak around $1410 \mathrm{~cm}^{-1}$ (as highlighted in Figure 1e). Therefore, for the construction of the sBW model, the Raman spectra of BW before and after melting were considered as two distinct excipients, while in case of $\mathrm{nBW}$ only one form of solid excipient was considered. 
a)

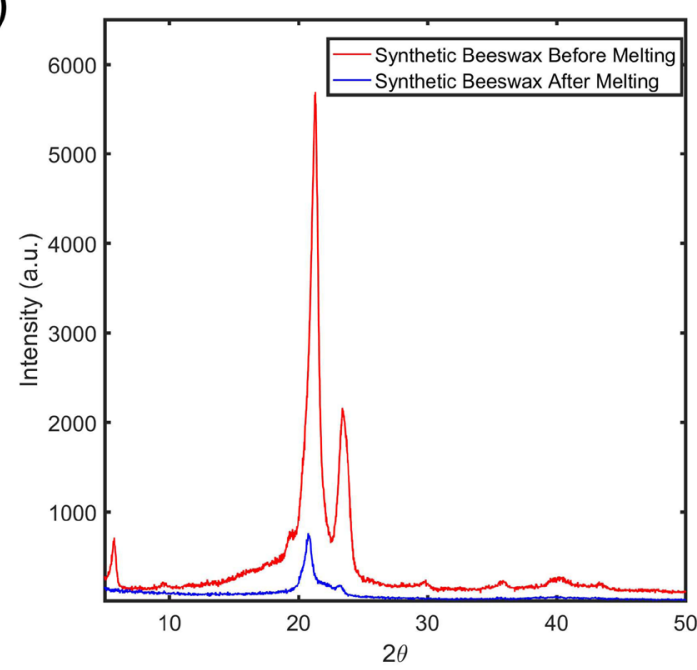

c)

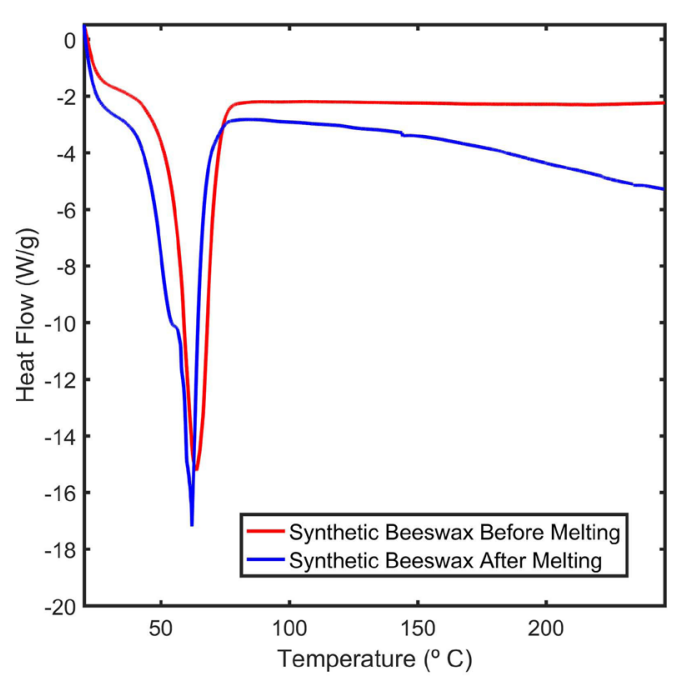

e)

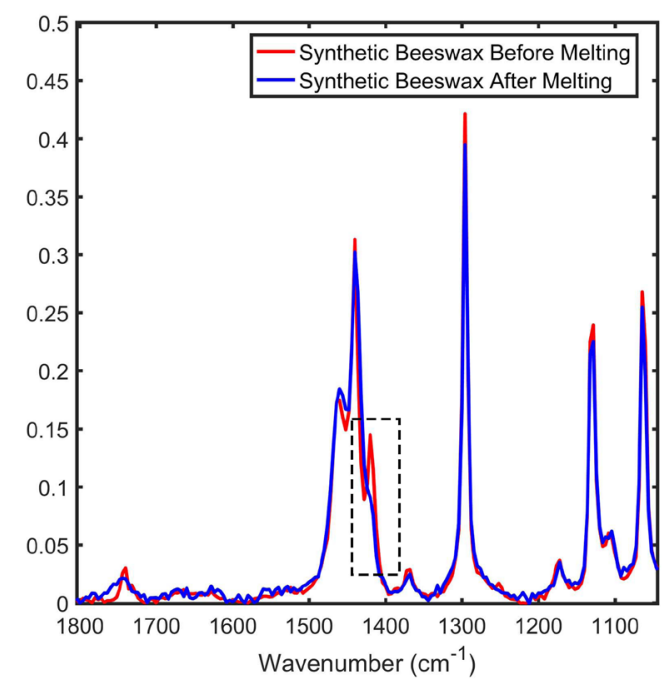

b)

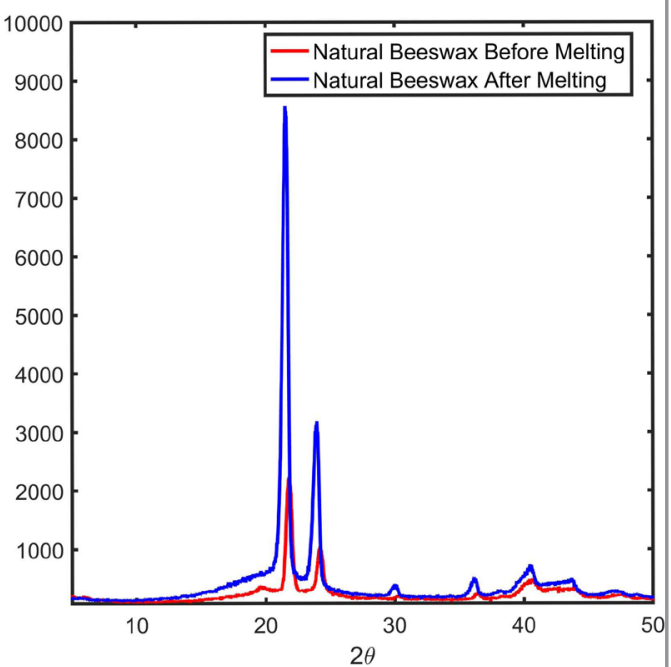

d)

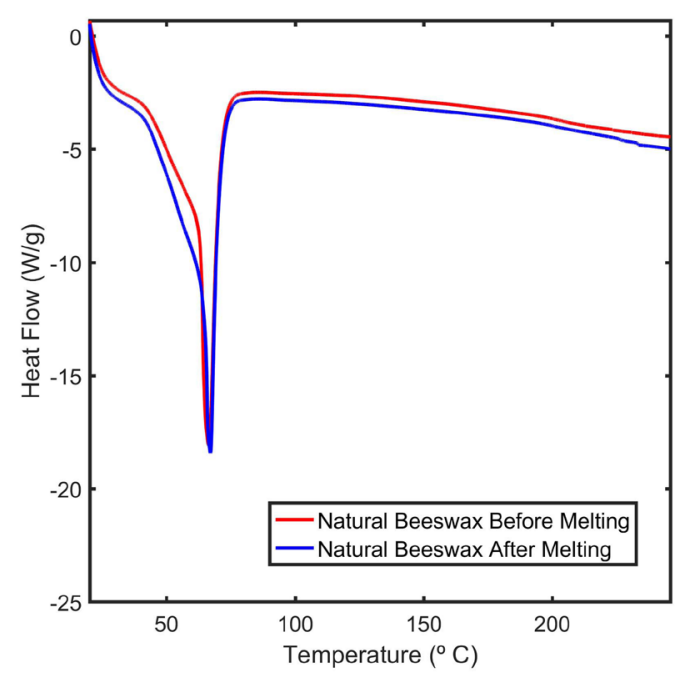

f)

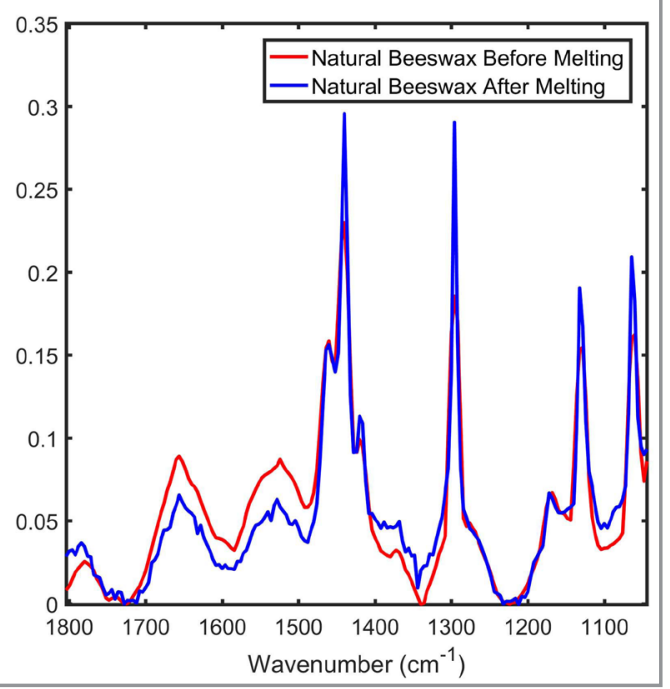

Figure 1. X-ray diffractograms of: (a) synthetic and (b) natural BW. DSC curves of: (c) synthetic and (d) natural BW. Raman spectra of: (e) synthetic and (f) natural BW. 


\section{Removal of Dead Pixels}

Before the use of MCR-ALS to build chemical maps, an initial analysis was made using CLS to detect defective pixels in the Raman images. Three examples are shown in Figure S1 for samples with $5.0 \%$ (w/w) copaiba oil at time zero (freshly prepared) and after 3 months, and for the $20.0 \%$ (w/w) copaiba oil sample, after 3 months.

In the first case (Figure S1a), some lines showed unusually high scores (red color) in the original dataset and because of this, the histogram displayed a tail on the left-hand side. In some situations these defective pixels could be replaced by the median of the neighbors [12]. In this dataset, however, this approach was not possible because some neighboring pixels were also defective. ICA could be a good tool to solve this problem. In this dataset there are only a few defective pixels, thus, 10 lines containing only these problematic pixels were created (entitled 'augmented defective spectra' in Figure S1a), with the objective to extract more easily these physical defects, as an independent component. Figure S1d shows the source signal of IC2 related to this phenomenon.

In the second and third cases (Figures S1b-S1c), it is clear that several pixels have defects, and thus, it is not necessary to use the strategy of augmenting with defective pixels, since ICA could be applied directly to these samples to isolate the problem of dead pixels. Figures S1d to S1f show that IC2 and IC4 are related to this problem, in the second and third cases, respectively. In other words, these ICs refer to physical problems in the images and are not due to a specific chemical compound.

After identification of the ICs containing information about dead pixels, Equations 2 and 3 were applied in Raman spectra. The chemical maps from CLS after this correction (Figures S1g-S1i) indicated that ICA was efficient in the correction.

\section{MCR-ALS Results of Natural Beeswax}

After ICA correction of these three samples, the chemical maps were created using MCR-ALS with an augmented matrix with all concentrations and times. In the case of nBW, the model converged in 15 iterations, the lack of fit (LOF) of PCA was $7.51 \%$ and the percent of explained variance was $96.96 \%$. Figure 2 shows the $\mathbf{S}$ matrix recovered (pure spectra recovered) and the original Raman spectra of excipients in these pre-formulations. Correlation coefficients were higher than 0.9302 , without rotational ambiguity in any of the recovered spectral profiles. It should be emphasized that, as the equality constraint was used for the copaiba oil spectrum, this profile did not suffer alterations and, therefore, its correlation coefficient was equal to 1 . Without the use of this constraint, rotational ambiguity occurred in the spectral region of 1320 to $1270 \mathrm{~cm}^{-1}$ and 1150 to $1050 \mathrm{~cm}^{-1}$ (Figure S2) resulting in a decrease of the correlation coefficient for the copaiba oil.

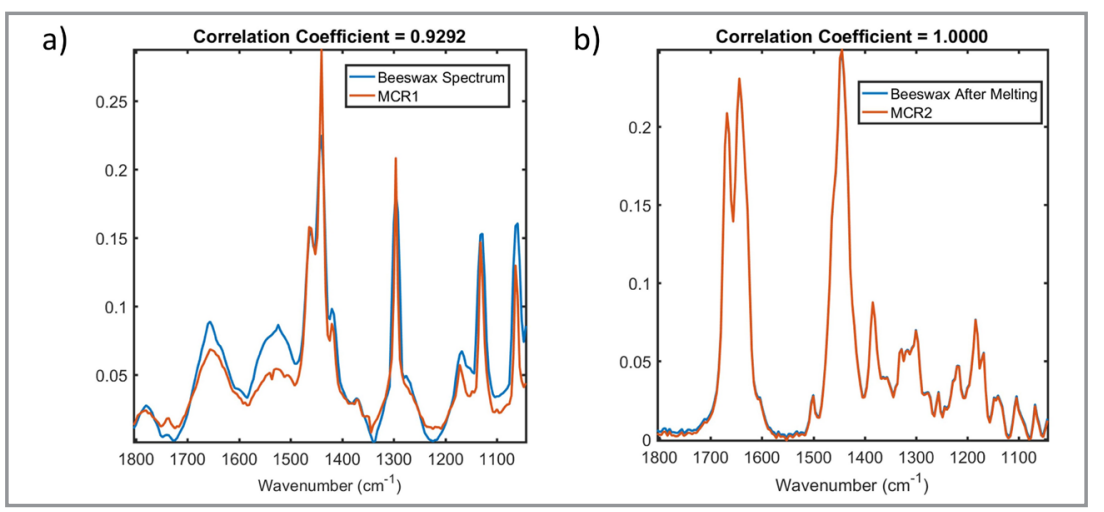

Figure 2. Original Raman spectra and spectral profile recovered by MCR-ALS, for nBW and copaiba oil pre-formulations.

Figure 3 shows the solid lipid maps and histograms obtained for these pre-formulations using nBW. However, the total number of chemical maps generated was high: for just one excipient, 10 maps and 
histograms were obtained (five concentrations at two different times) giving a total of 20 Raman maps for the two lipids. This large volume of data makes the interpretation difficult, and for this reason, the experimental concentrations were plotted against the mean values of the scores, along with the standard deviations of the histograms (Figure 4).

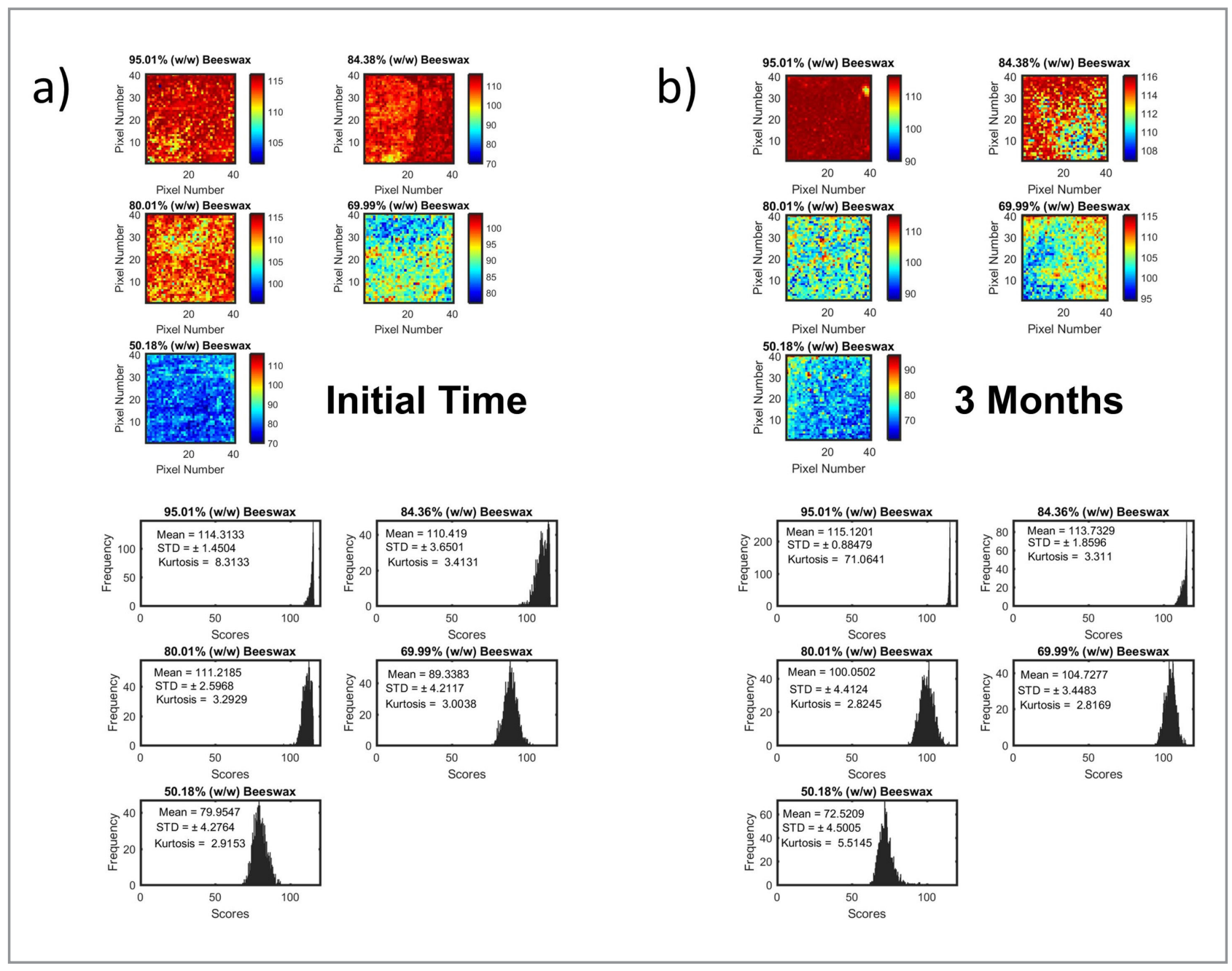

Figure 3. MCR-ALS score maps for nBW excipient a) at initial time and b) after 3 months.
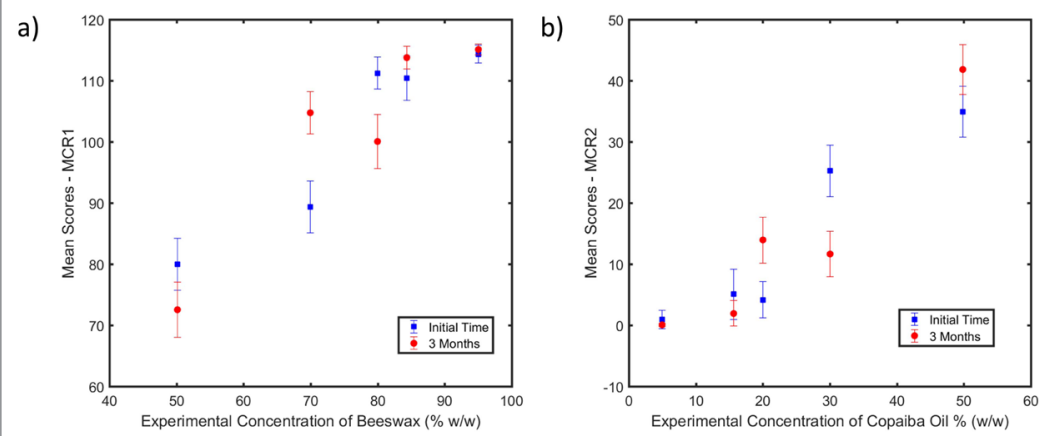

Figure 4. STD and mean of MCR-ALS scores for: a) nBW and b) copaiba oil. 
As can be seen in Figure 4, there is no tendency to differentiate the samples in relation to time, i.e., samples of the initial time have sometimes higher and sometimes lower mean values than samples after 3 months. However, it is interesting to note that the differences between the samples (initial vs. 3 months) generally tend to be higher as the amount of the liquid excipient increases, indicating that more stable samples are produced with lower amounts of the liquid lipid. In addition to visual inspection of maps, the $\mathrm{DHI}$ and standard deviation of the histograms (STD) were used for evaluation of the results, as shown in Table I. As reported by Ma et al. [24] and Farkas, Nagy and Marosi [23], DHI is a suitable tool to evaluate the miscibility of components in chemical images. However, its use alone is not recommended because sometimes it cannot represent the distributional homogeneity and, therefore, it is also interesting to evaluate the images associated with the STD of the corresponding histograms. Farkas, Nagy and Marosi [23] also suggested one modification, achieved by weighting the scores and relative standard deviations.

Table I. STD and DHI obtained for samples developed using nBW

\begin{tabular}{lccccc}
\hline & & \multicolumn{2}{c}{ nBW } & \multicolumn{2}{c}{ Copaiba Oil } \\
\hline Time & $\begin{array}{c}\text { Concentration of } \\
\text { copaiba oil } \\
(\% \text { w/w })\end{array}$ & STD & DHI & STD & DHI \\
\hline \multirow{4}{*}{ Initial } & 5.0 & 1.4 & 2.8 & 1.5 & 3.5 \\
& 15.6 & 3.6 & 4.7 & 4.1 & 4.7 \\
& 20.0 & 2.6 & 2.7 & 2.9 & 2.9 \\
& 30.0 & 4.2 & 4.0 & 4.2 & 3.8 \\
After 3 & 49.8 & 4.3 & 3.2 & 4.2 & 3.2 \\
Months & 5.0 & 0.9 & 2.2 & 0.3 & 1.8 \\
& 15.6 & 1.9 & 4.5 & 2.1 & 4.7 \\
& 20.0 & 4.4 & 1.8 & 3.8 & 4.3 \\
& 30.0 & 3.5 & 4.3 & 3.7 & 4.3 \\
\hline
\end{tabular}

The results shown in Figure 4 and Table I indicate that copaiba oil does not have miscibility problems with the $\mathrm{nBW}$, as low STD and DHI were observed. The standard deviations (STD) varied between 0.3 to 4.5 and $\mathrm{DHI}$ from 1.8 to 4.7 .

Another factor to be evaluated is that there is no difference between the STD and DHI values in relation to the time of the samples. This implies no alterations of formulations/phase separation in natural BW preformulations over the evaluated time period (3 months).

\section{MCR-ALS Results of Synthetic Beeswax}

The model for formulations using sBW converged after 63 iterations, with lack of fit (LOF) in Principal Component Analysis (PCA) of $0.39 \%$ and $99.31 \%$ of the explained variance. Figure 5 shows $\mathbf{S}_{1}$ (spectral profile recovered by MCR-ALS) and original Raman spectra of excipients. There were no problems of rotational ambiguity, that is, the peak due to one compound was not recovered in the spectrum of another. In addition, the correlation coefficients were above 0.9743 . The spectral profile recovered for copaiba oil (Figure 5c) had a signal intensity lower than the those of the beeswaxes, therefore the $y$-axis was changed. In this case, three profiles were recovered, since sBW showed spectral differences before and after melting, especially in the region of $1400-1500 \mathrm{~cm}^{-1}$. 

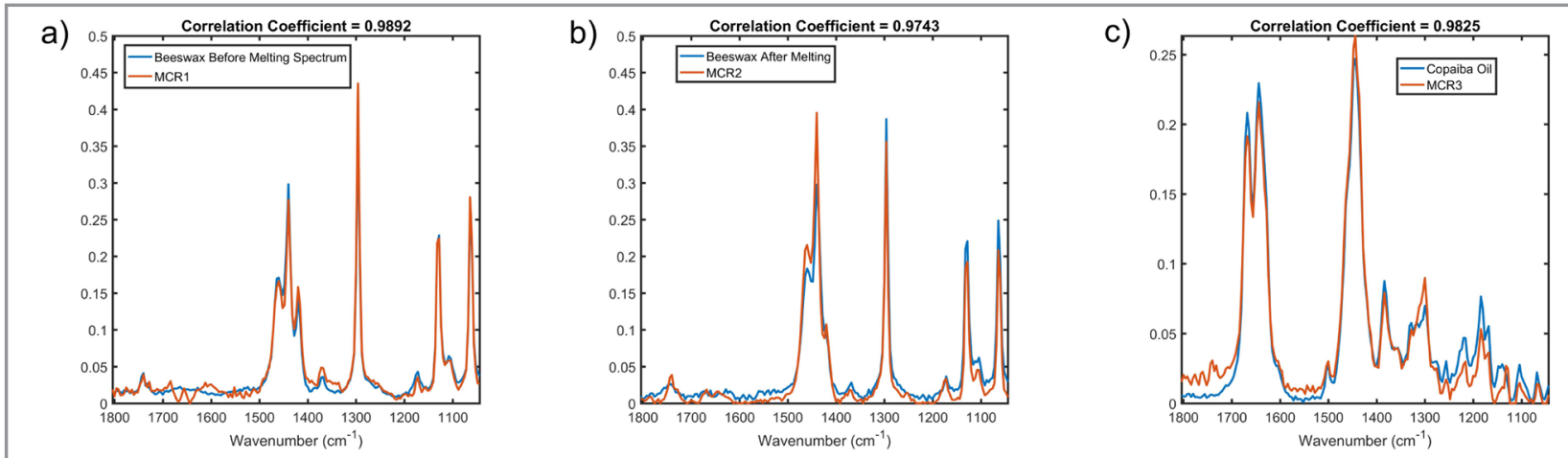

Figure 5. Original Raman spectra and spectral profiles recovered by MCR-ALS (see text) for SBW and copaiba oil formulations.

The histograms and chemical maps built using MCR-ALS scores for sBW before melting are shown in Figure S3. Table II shows the STD and DHI values obtained for each excipient. As can be seen, the standard deviations (STD) and DHI values tend to be slightly higher than those observed for nBW. Copaiba oil did not have miscibility problems with two forms of synthetic beeswax, with the low STD and DHI values.

Figure 6 displays the plot of experimental concentrations against the mean values of the scores, along with the standard deviations of the histograms. As observed in Figure 6a, the mean values of the images at the initial times are lower than in the images acquired after 3 months. On the other hand, in the case of the sBW form found after melting (Figure 6b), the inverse occurs: the values at the initial time are higher than in the other times. This tendency did not appear in case of copaiba oil. Therefore, a polymorphic transformation is probably taking place in the sBW over time, in which the form that appeared after the melting process is converting back into the initial form.

Moreover, the sample with the highest amount of BW (90\%) showed smaller difference between the initial time and after 3 months, similar to the results obtained for the $\mathrm{nBW}$. Thus, one assumption is that the presence of liquid excipient caused changes in the mobility of the structures of the solid excipient, with a greater impact at higher concentrations. This can also be one of the explanations for the better performance of NLC formulations at lower concentrations of the liquid lipid $[1,25]$.

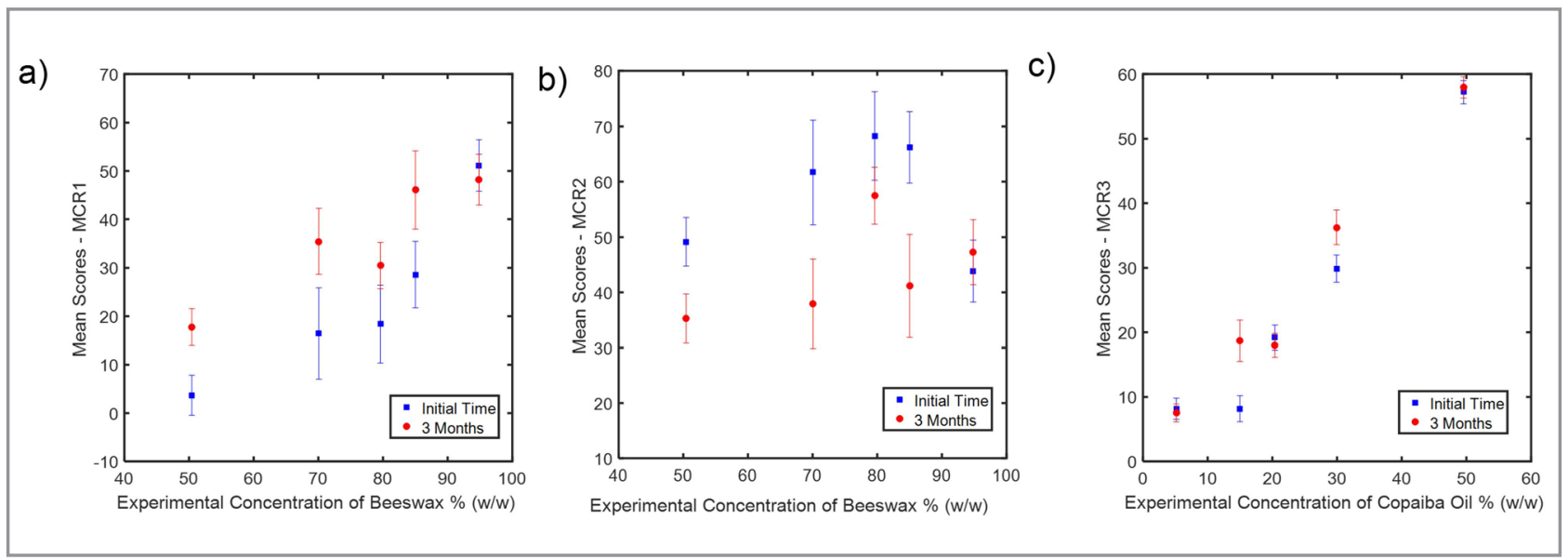

Figure 6. STD and mean of MCR-ALS scores for: a) sBW before melting, b) sBW after melting and c) copaiba oil for formulations developed using synthetic wax. 
Table II. STD and DHI obtained for samples developed using sBW

\begin{tabular}{lccccccc}
\hline & \multicolumn{3}{c}{ sBW Before Melting } & \multicolumn{2}{c}{ sBW After Melting } & \multicolumn{2}{c}{ Copaiba Oil } \\
\hline Time & $\begin{array}{c}\text { Concentration of } \\
\text { copaiba oil } \\
(\% \text { w/w) }\end{array}$ & STD & DHI & STD & DHI & STD & DHI \\
\hline \multirow{4}{*}{ Initial } & 5.1 & 5.3 & 2.6 & 5.6 & 2.6 & 1.6 & 3.4 \\
& 15.0 & 6.9 & 4.4 & 6.5 & 4.1 & 2.0 & 4.1 \\
& 20.4 & 8.0 & 5.7 & 8.0 & 5.1 & 1.9 & 2.5 \\
& 29.9 & 9.4 & 5.1 & 9.4 & 4.8 & 2.1 & 2.1 \\
After 3 & 49.6 & 4.1 & 3.2 & 4.3 & 1.9 & 1.8 & 4.9 \\
Months & 5.1 & 5.2 & 4.2 & 5.9 & 4.1 & 1.4 & 2.9 \\
& 15.0 & 8.1 & 5.2 & 9.3 & 5.0 & 3.2 & 3.7 \\
& 20.4 & 4.8 & 3.8 & 5.1 & 3.3 & 1.8 & 4.1 \\
& 29.9 & 6.8 & 4.3 & 8.1 & 4.2 & 2.6 & 2.9 \\
\hline
\end{tabular}

Comparing the STD and DHI values of sBW with those of $\mathrm{nBW}$, sBW have higher values than $\mathrm{nBW}$. However, copaiba oil STD and DHI are similar in both cases, indicating this difference is due to the two polymorphic forms. Moreover, the homogeneity of the formulations does not seem to change significantly over the time (i.e., neither the STD and DHI increased significantly nor did two distributions appear in the histograms).

\section{CONCLUSIONS}

A structural change was identified in the synthetic BW after melting, which was not observed for the natural BW using DSC, DRX and Raman techniques. But the miscibility of natural and synthetic BW with copaiba oil was not significantly different, based on the STD and DHI values. Nevertheless, the form that appeared after melting of sBW, seems to transform back to the initial form (Figures 6a,b).

It was also observed that the differences between freshly prepared and 3 months samples tended to be higher as the amount of the liquid lipid increased in the pre-formulation. This influence of the liquid excipient in solid materials has also been found in other works $[26,27]$ which makes it even more important to analyze the various properties of these mixtures of solid and liquid excipients, as a function of their concentrations, miscibility and physical changes.

\section{Conflicts of interest}

The authors declare they have no conflict of interest.

\section{Acknowledgements}

The authors thank Fundação de Amparo à Pesquisa do Estado de São Paulo - FAPESP [2016/05636-9], Conselho Nacional de Desenvolvimento Científico e Tecnológico - CNPq [H.M. scholarship - 142441/20155], Coordenação de Aperfeiçoamento de Pessoal de Nível Superior - CAPES [Finance Code 001] and Instituto Nacional de Ciência e Tecnologia em Bioanalítica - INCT-Bioanalitica [FAPESP/INCT 14/508673; CNPq/INCT 465389/2014-7] for financial support; GM Ceras and Megh Indústria e Comércio for the donation of beeswaxes. 


\section{REFERENCES}

1. Ribeiro, L. N. M.; Breitkreitz, M. C.; Guilherme, V. A.; da Silva, G. H. R.; Couto, V. M.; Castro, S. R.; de Paula, B. O.; Machado, D.; de Paula, E. Eur. J. Pharm. Sci., 2017, 106, pp 102-112 (http://dx.doi. org/10.1016/j.ejps.2017.05.060).

2. Attama, A. A.; Schicke, B. C.; Müller-Goymann, C. C. J. Drug Deliv. Sci. Technol., 2007, 17, pp 103112 (http://dx.doi.org/10.1016/S1773-2247(07)50016-X).

3. Fratini, F.; Cilia, G.; Turchi, B.; Felicioli, A. Asian Pac. J. Trop. Med., 2016, 9, pp 839-843 (https://doi. org/10.1016/j.apjtm.2016.07.003).

4. Environmental Protection Agency. Beeswax, synthetic. 2020. Available from: https:// ofmpub.epa.gov/sor_internet/registry/substreg/searchandretrieve/substancesearch/search. do?details=displayDetails\&selectedSubstanceld=6927 [Accessed 18 January 2021].

5. Dias, D. D. O.; Colombo, M.; Kelmann, R. G.; Kaiser, S.; Lucca, L. G.; Teixeira, H. F.; Limberger, R. P.; Veiga, V. F.; Koester, L. S. Ind. Crop. Prod., 2014, 59, pp 154-162 (https://doi.org/10.1016/j. indcrop.2014.05.007).

6. Reátegui, J. L. P.; Fernandes, F. P.; dos Santos, P.; Rezende, C. A.; Sartoratto, A.; Queiroga, C. L.; Martínez, J. J. Supercrit. Fluid, 2018, 140, pp 364-371 (https://doi.org/10.1016/j.supflu.2018.07.021).

7. Da Trindade, R.; da Silva, J. K.; Setzer, W. N. Int. J. Mol. Sci. 2018, 19 (5), 1511 (https://doi.org/10.3390/ ijms19051511).

8. Attama, A. A.; Schicke, B. C.; Müller-Goymannb, C. C. Eur. J. Pharm. Biopharm., 2006, 64 (3), pp 294-306 (https://doi.org/10.1016/j.ejpb.2006.06.010).

9. Dantas, I. L.; Bastos, K. T. S.; Machado, M.; Galvão, J. G.; Lima, A. D.; Gonsalves, J. K. M. C.; Almeida, E. D. P.; Araújo, A. A. S.; de Meneses, C. T.; Sarmento, V. H. V.; et al. J. Therm. Anal. Calorim., 2018, 132, pp 1557-1566 (https://doi.org/10.1007/s10973-018-7072-7).

10. Gaillard, Y.; Mija, A.; Burr, A.; Darque-Ceretti, E.; Felder, E.; Sbirrazzuoli, N. Thermochim. Acta, 2011, 521, pp 90-97 (https://doi.org/10.1016/j.tca.2011.04.010).

11. Sacré, P.-Y. Y.; Lebrun, P.; Chavez, P.-F. F.; Bleye, C. De; Netchacovitch, L.; Rozet, E.; Klinkenberg, R.; Streel, B.; Hubert, P.; Ziemons, E. Anal. Chim. Acta, 2014, 818, pp 7-14 (https://doi.org/10.1016/j. aca.2014.02.014).

12. Vidal, M.; Amigo, J. M. Chemom. Intell. Lab. Syst., 2012, 117, pp 138-148 (https://doi.org/10.1016/j. chemolab.2012.05.009).

13. Rutledge, D. N.; Bouveresse, D. J-R. TrAC Trends Anal. Chem., 2013, 50, pp $22-32$ (https://doi. org/10.1016/j.trac.2013.03.013).

14. Rutledge, D. N.; Bouveresse, D. J-R. TrAC Trends Anal. Chem., 2015, 67, pp 220 (https://doi. org/10.1016/j.trac.2015.02.001).

15. International Council for Harmonisation of Technical Requirements for Pharmaceuticals for Human Use - ICH; Q1A(R2). Stability testing of new drug substances and products. Brussels, ICH, 2003.

16. Sabin, G. P.; de Souza, A. M.; Breitkreitz, M. C.; Poppi, R. J. Quim. Nova, 2012, 35, pp 612-615 (https://doi.org/10.1590/S0100-40422012000300030).

17. Mazet, V.; Carteret, C.; Brie, D.; Idier, J.; Humbert, B. Chemom. Intell. Lab. Syst., 2005, 76, pp 121133 (https://doi.org/10.1016/j.chemolab.2004.10.003).

18. Jutten, C.; Herault, J. Signal Processing, 1991, 24, pp 1-10 (https://doi.org/10.1016/01651684(91)90079-X).

19. Cardoso, J. F.; Souloumiac, A. IEE Proceedings, Part F Radar Signal Process, 1993, 140, pp 362-370 (https://doi.org/10.1049/ip-f-2.1993.0054).

20. Bouveresse, D. J-R.; Rutledge, D. N. Independent Components Analysis: Theory and Applications. In: Ruckebusch, C. (Ed.). Resolving Spectral Mixtures with Applications from Ultrafast Time-Resolved Spectroscopy to Super-Resolution Imaging, v. 30 of Data Handling in Science and Technology. Elsevier, [s.l.], 2016, pp 225-277 (https://doi.org/10.1016/B978-0-444-63638-6.00007-3). 
21. Bouveresse, D. J-R.; Moya-González, A.; Ammari, F.; Rutledge, D. N. Chemom. Intell. Lab. Syst., 2012, 112, pp 24-32 (https://doi.org/10.1016/j.chemolab.2011.12.005).

22. Jaumot, J.; de Juan, A.; Tauler, R. Chemom. Intell. Lab. Syst., 2015, 140, pp 1-12 (https://doi. org/10.1016/j.chemolab.2014.10.003).

23. Farkas, A.; Nagy, B.; Marosi, G. Period. Polytech. Chem. Eng., 2017, 62, pp 1-7 (https://doi.org/10.3311/ PPch.10143).

24. Ma, L.; Zhou, L.; Xu, M.; Huang, X.; Zhang, Q.; Dai, S.; Qiao, Y.; Wu, Z. Spectrochim. Acta - Part A Mol. Biomol. Spectrosc., 2018, 204, pp 783-790 (https://doi.org/10.1016/j.saa.2018.06.081).

25. da Silva, G. H. R.; Ribeiro, L. N. M.; Mitsutake, H.; Guilherme, V. A.; Castro, S. R.; Poppi, R. J.; Breitkreitz, M. C.; de Paula, E. Int. J. Pharm., 2017, 529, pp 253-263 (https://doi.org/10.1016/j. ijpharm.2017.06.066).

26. Mitsutake, H.; Castro, S. R.; De Paula, E.; Poppi, R. J.; Rutledge, D. N. Int. J. Pharm., 2018, 552, pp 119-129 (https://doi.org/10.1016/j.ijpharm.2018.09.058).

27. Unga, J.; Matsson, P.; Mahlin, D. Int. J. Pharm., 2010, 386, pp 61-70 (https://doi.org/10.1016/j. ijpharm.2009.10.049). 


\section{SUPPLEMENTARY MATERIAL}

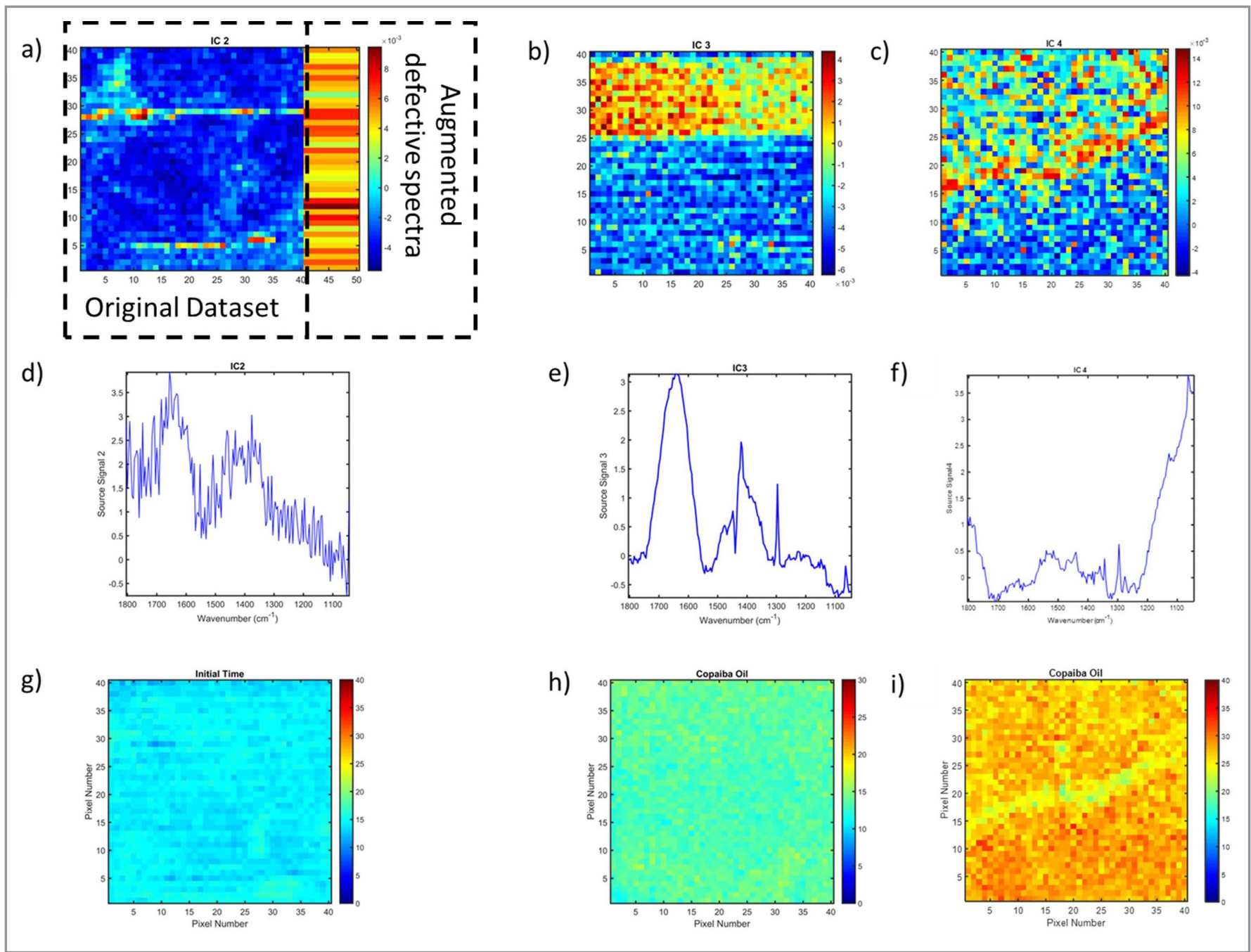

Figure S1. Chemical maps obtained by ICA before correction for samples: (a) 5.0\% (w/w) copaiba oil at time zero (freshly prepared), (b) $5.0 \%$ (w/w) copaiba oil after 3 months, and (c) for the $20.0 \%$ (w/w) copaiba oil sample, after 3 months. Source signals: (d) IC2 for 5.0\% (w/w) copaiba oil at time zero (freshly prepared), (e) IC3 for 5.0\% (w/w) copaiba oil after 3 months, and (f) IC4 for the $20.0 \%$ (w/w) copaiba oil sample, after 3 months. Chemical maps obtained by CLS after correction for samples: (g) 5.0\% (w/w) copaiba oil at time zero (freshly prepared), (h) $5.0 \%$ $(\mathrm{w} / \mathrm{w})$ copaiba oil after 3 months, and (i) for the $20.0 \%$ (w/w) copaiba oil sample, after 3 months. 
a)

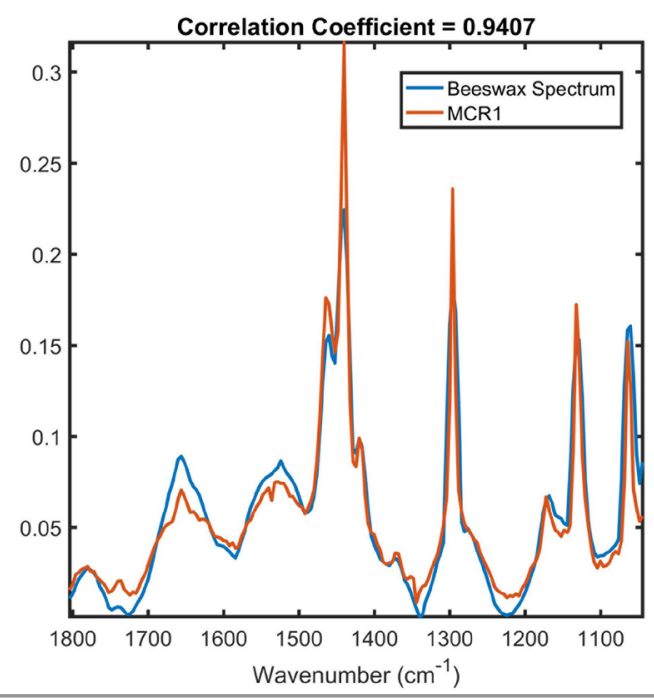

b)

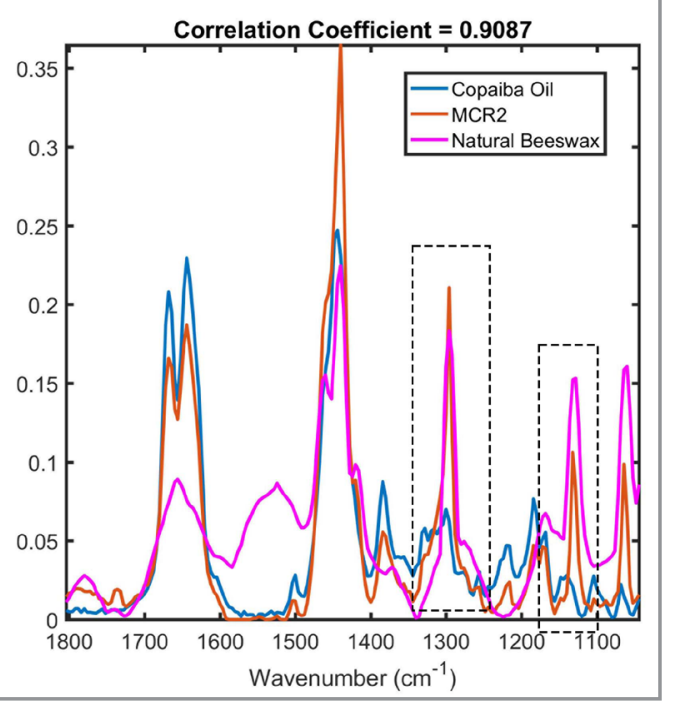

Figure S2. Original Raman spectra and spectral profile recovered by MCR-ALS, for nBW and copaiba oil pre-formulations without equality constraint.



Figure S3. Histograms and chemical maps built using MCR-ALS scores for SBW before melting. 